\title{
Design Considerations of Ultra Dense $5 G$ Network in Millimetre Wave Band
}

\author{
Naser Al-Falahy ${ }^{(1),(2)}$ and Omar Y. K. Alani ${ }^{(1)}$ \\ (1) University of Salford, Manchester, United Kingdom \\ (2) University of Anbar, Iraq \\ N.F.A.AL-Falahy@edu.salford.ac.uk, O.Y.K.Alani@salford.ac.uk
}

\begin{abstract}
The fifth Generation (5G) network will provide services with extreme data rate and latency demands compared to current cellular networks, and provide massive capacity and connectivity to multitude of devices with diverse requirements and applications. In this paper, dense deployment of small cells in high carrier frequency is considered as the theme of future $5 \mathrm{G}$ network. Network densification depicted in this work includes densification over the frequency by the adoption of wider bandwidth in the millimetre wave band, and densification over the space through higher number of antennas, higher sectorisation order, and dense deployment of small cells. The reference signal received power (RSRP) and quality (RSRQ), and signal to interference plus noise ratio (SINR) have been considered as the metrics for the design evaluation. Our results show that network densification has significant importance in improving data rate to meet $5 \mathrm{G}$ vision. And that dense deployment of small cells has better performance over higher sectorisation order, due to the higher line of site coverage and lower interference in the former case. In addition, the results show that densification in term of increasing the antennas is also vital to enable spatial multiplexing through multiinput-multi-output and enable beamforming to improve SINR, which eventually improve the data rate. Foliage loss and rain at millimetre wave bands are significant, and therefore, their impact has been evaluated as well.
\end{abstract}

Keywords-5G network modelling; millimetre wave; network densification; rain attenuation; beamforming.

\section{INTRODUCTION}

Due to the significant development and the huge use of data hungry devices such as mobile phones, tablets, and laptop, Global Mobile Data Traffic (GMDT) is anticipated to surge to 30.6 exabytes per month by 2020 . Mobile data traffic will grow at an annual growth rate of 53\% from 2015 to 2020 [1].

The spectrum is becoming too scarce at the microwave bands as the overwhelming majority of communication systems are operating on $<3 \mathrm{GHz}$ band. Therefore, there is a need to move to the millimetre wave ( $\mathrm{mm}$-wave) bands as there is a very wide of unexploited spectrum ranging from 20 to $90 \mathrm{GHz}$. This band is a potential and essential bandwidth for The fifth Generation (5G) system for achieving 1000 folds capacity increase over Long Term Evolution (LTE) [2][3]. The mm-wave spectrum is the extremely high frequency (EHF) band ranging from $30 \mathrm{GHz}$ to $300 \mathrm{GHz}$. While $3-30 \mathrm{GHz}$ is known as the super high frequency (SHF) band. And because SHF and EHF has similar propagation characteristics, the $3-300 \mathrm{GHz}$ band is collectively named millimetre-wave frequency bands that have wavelengths from 1 to 100 millimetre [4].

In this context, the data throughput per user can increase according to many factors, the fundamental equation is [5]:

$$
R=m(B / n) \log _{2}(1+\operatorname{SINR})
$$

where $R$ is the data throughput, $\mathrm{m}$ is the number of spatial links between station and user, B is the signal bandwidth, $\mathrm{n}$ is the number of users connected to a base station, and SINR is the received signal power, to interference plus noise ratio.

As clarified in eq.(1), the bandwidth $B$ can be increased through the use of wider bandwidth at millimetre wave band, which will directly increasing the capacity. And the loading factor (n) can be decreased by increasing sectorisation order per site and deploying denser small cells base stations. Spatial Streams (m) can be increased through increasing the number of antennas at the station end and user end, which is known as Multi-InputMulti Output (MIMO).

Ultra-Dense Network (UDN) can increase signal Power (S) by bringing the access point closer to the user. However, this will also increase the interference (I) from nearby sectors and sites.

Densification has been envisioned as the future theme of the next generation wireless network in [5] and the key mechanism for wireless evolution over the next decade. While in [6], the authors study the relation between network capacity and the density of micro base stations in HetNets. In [7], a new mobility-enhanced scheme is designed and analysed to improve handover in UDN, as mobility enhancement is vital in this approach. Resource management has been extensively studied and discussed in [8], while in [9], a novel framework for indoor planning of mm-waves UDN has been introduced. The analysis is based on multi-objective optimization and ray-tracing techniques to provide a multi Gbps data throughput. Densification in $73 \mathrm{GHz}$ carrier has been studied in [10], the authors have presented and validated a new blockage model, and they prove that mm-wave UDN is the key to achieve both better coverage and data rates. 
In this paper, we have introduced UDN based mm-wave, particularly in $28 \mathrm{GHz}$ carrier. Densification has been represented by: higher order sectorisation per site [11], higher number of antennas per cell, and denser small cells deployments per unit area. In addition UDN has been used in this work to compensate for the severe attenuation in heavy rainfall. And due to increased interference in UDN, beamforming (BF) has been used to increase signal path gain and to suppress interference in UDN environment.

The rest of the paper is organised as follow: section II presents the densification in 5G. The network model has been introduced in section III, followed by the UDN results and their discussion. Section IV presents the BF in mm-wave, followed by their results and discussion. Finally, conclusions are drawn in section V.

\section{NETWORK DENSIFICATION IN 5G}

The extremely high data throughput and the very low latency required by $5 \mathrm{G}$ can only be satisfied through network densification. Densification over both frequency and space are necessary to fulfil $5 \mathrm{G}$ vision in term of (i) massive capacity: increases on the order of several magnitudes. The future requirement is a 1000x increase in data traffic for 2020 and beyond. (ii) Increased indoor and small cells/hotspot traffic: will be the majority of mobile traffic volume. Today, about 70 percent of mobile traffic happens indoors; in the future, indoor data traffic as well as hotspot areas may exceed this figure. (iii) Higher numbers of connected devices (massive connectivity): stemmed from Internet of Things (IoT) due to massive machineto-machine (M2M) communications and applications, as all devices that benefit from internet connectivity will become connected. (iv) Latency: latency reductions become vital since technologies such as D2D, M2M, tactile internet, and cloudbased applications, require very short end-to-end latency. All these improvements are essential for the Quality of Experience (QoE) provision to huge number of users in $5 \mathrm{G}$ era.

\section{NETWORK MODEL}

The simulation has been carried out by ICS Designer by ATDI, a high-fidelity ray-tracing solvers for radio network planning and optimisation [12]. The inter site distance (ISD) has been selected from 600 to $200 \mathrm{~m}$ (see fig. 1 left) to simulate different scenario of dense deployment based on recent contribution [13]. Higher sectorisation order has been model as well as shown in fig. 1 right, where $3,4,6,8$, and 10 sectors has been modelled to evaluate their performance. Furthermore, user equipment (UE) distribution is random and outdoor in city streets and open areas, and the network shares its resources among users according to the fairness of the scheduler and data rate flow. The basestation height is $10 \mathrm{~m}$ above ground and distributed outdoor in street corners. The $28 \mathrm{GHz}$ has been selected for the carrier frequency with $500 \mathrm{MHz}$ bandwidth. MIMO and orthogonal frequency division multiplexing (OFDM) have superior performance and therefore they were selected in this work as the future $5 \mathrm{G}$ multiplexing scheme. Details of the simulation parameters are shown in table I.
The wide bandwidth is essential to provide the resources required for supporting future user demands, wider bandwidth of up to $1 \mathrm{GHz}$ is possible in this band, and however, it could be fragmented. More bandwidth can be used when increasing the carrier frequency to the $60 \mathrm{GHz}$ the free-licensed band where $7 \mathrm{GHz}$ bandwidth (57-64) $\mathrm{GHz}$ is available, limited with high oxygen attenuation. The E-band has much wider bandwidth of $12.9 \mathrm{GHz}$ located at $(71-76,81-86,92-95) \mathrm{GHz}$. The $28 \mathrm{GHz}$ band has the favourable propagation condition among these bands as shown in fig. 2 .



Fig.1 Network model, left dense deployment, right- eight sectorisation order

\begin{tabular}{|c|c|}
\hline \multicolumn{2}{|c|}{ NETWORK MODEL PARAMETERS } \\
\hline Model parameter & Value \\
\hline Channel Type & Downlink \\
\hline Tx Power & $3 \mathrm{~W}$ \\
\hline Tx antenna gain & $15 \mathrm{~dB}$ \\
\hline $\mathrm{Rx}$ antenna gain & $3 \mathrm{~dB}$ \\
\hline Tx beamwidth (HPBW) & $\begin{array}{l}60^{\circ}(3,4,6) \text { sectors, } 20^{\circ}(8,10) \text { sectors } \\
\text { with } 7^{\circ} \text { vertical Beamwidth }\end{array}$ \\
\hline Rx beamwidth & Omni-directional, Directional (BF) \\
\hline Carrier frequency & $28 \mathrm{GHz}$ \\
\hline Bandwidth & $500 \mathrm{MHz}$ \\
\hline Antenna Type & Standard (SISO) and MIMO \\
\hline Tx Antenna height & $10 \mathrm{~m}$ \\
\hline Rx antenna height & $1.5 \mathrm{~m}$ \\
\hline Polarisation & Vertical \\
\hline Modulation & Adaptive (QPSK, 16QAM, 64QAM) \\
\hline Scheduler & Proportional Fair \\
\hline Downlink shared channels & $70 \%$ (resources) \\
\hline Downlink control channels & $30 \%$ (overhead) \\
\hline No. of $\mathrm{Rx}$ & 600 (random, outdoor) \\
\hline Area of steady & $1 \mathrm{~km}^{2}$ \\
\hline Noise Figure & $10 \mathrm{~dB}$ \\
\hline Noise Density & $-174 \mathrm{dBm} / \mathrm{Hz}$ \\
\hline Number of sites & 5,10 , and 20 \\
\hline Sectorisation order & $3,4,6,8$, and 10 \\
\hline ISD & 600,400 , and $200 \mathrm{~m}$ \\
\hline Beamforming (BF) & Yes (Rx BF, Tx BF) \\
\hline Rain (ITU-R 838 model) & $(0,0.25,2.5,10,25,50,150) \mathrm{mm} / \mathrm{h}$ \\
\hline Traffic model & Full buffer \\
\hline
\end{tabular}

\section{A. Densification over Frequency (in Millimetre wave)}

It is widely accepted that mm-wave need to be used with very limited cell radius $<200 \mathrm{~m}$, to minimize high pathloss at this band (by bringing the access point closer to the user which reduce signal path). Fortunately, this action fits well with the trend of dense deployment of small cells [4]. Using millimetre wave for cellular purposes face limiting factors such as: 
1- Foliage Losses: Foliage losses at millimetre wave frequencies are very high and a point of concern. An empirical formulae was developed by Marcus and Pattan in [14]. They address the foliage loss by:

$$
L=0.2 f^{0.3} R^{0.6}
$$

where $\mathrm{L}$ is the foliage loss in $\mathrm{dB}$, f: frequency in $\mathrm{MHz}$; $\mathrm{R}$ : depth of foliage in metres, and applies for $\mathrm{R}<400 \mathrm{~m}$ and applied for (20-95) $\mathrm{GHz}$ frequencies. A significant loss in tens of $\mathrm{dB}$ can occur when the foliage depth increase which can become a limiting factor due to the severe fading.

2- Rain Attenuation: Millimetre-wave can suffer very high attenuations in the case of heavy rain. Since their wavelengths are approximately the same size as the rain drop and therefore cause high signal scattering. Heavy rain can cause severe attenuation and jeopardise communication at these frequencies, the higher the frequency the worst the attenuation. As we will see in this paper, minimising inter-site-distance (ISD) can minimise the rain attenuation (shown in fig.2) where 28, 60, 72, $82 \mathrm{GHz}$ bands are compared. It is clearly that $28 \mathrm{GHz}$ band has the lowest attenuation due to their longer wavelengths compared with rain drop.

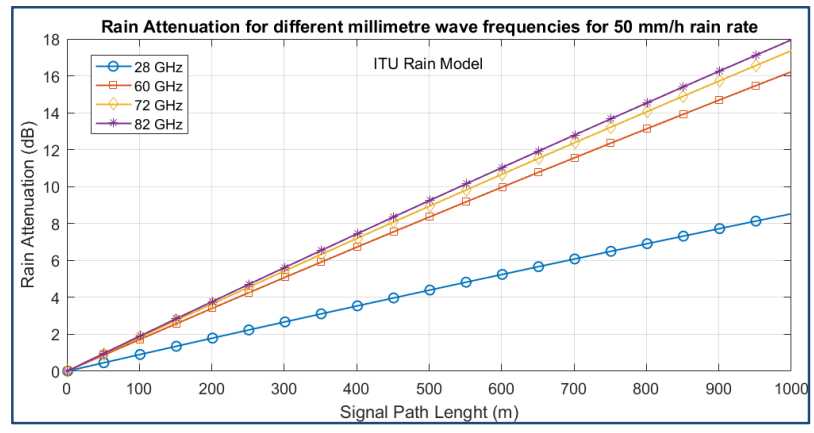

Fig.2 Rain attenuation vs. distance for $28,60,72,82 \mathrm{GHz}$ bands

Beside, heavy rain occurred only in limited time and in certain geographical areas. However, when mm-wave network is disrupted by heavy rain, a backup cellular network should cover these areas to maintain network availability with acceptable quality of service (QoS).

3- High penetration and Path Loss: One of the challenges in mm-wave band is the high pathloss compared to microwave bands below $3 \mathrm{GHz}$, which make it too sensitive to blockage such as buildings. Generally, the pathloss equation is [15]:

$$
L_{F S}=32.4+20 \log _{10} f+20 \log _{10} R
$$

where $\mathrm{L}_{\mathrm{FS}}$ is the pathloss in $\mathrm{dB}, f$ is the frequency in $\mathrm{GHz}$, and $R$ is the distance between the base station and the user in metre. Therefore there will be around $10 \mathrm{~dB}$ of extra pathloss when moving the operating frequency from $2.8 \mathrm{GHz}$ to $28 \mathrm{GHz}$.

\section{B. Densification over Space}

\section{Multi antennas in millimetre wave}

Standard -single input single output- SISO and MIMO antennas have been used for both base stations and user equipment (UE's). MIMO has a superior performance over standard antennas in term of reference signal received power (RSRP) and can provide better SINR to users as shown in fig.3, where cumulative distribution function (CDF) and probability distribution function (PDF) have been used for evaluation representation. CDF clearly shows the significant improvement of using more antennas over the standard antennas. In addition, MIMO can support higher data throughput through spatial multiplexing. Spatial multiplexing (SM) - even in line-of-site is likely to be provided by certain antennas separation [16] as high separation in term of wavelength $\lambda$ will be feasible (few millimetres) when we use millimetre wave band. In addition, distributed MIMO can ensure unique uncorrelated paths for SMMIMO operation in line of site (LOS) environment.

\section{Increasing sectorisation order}

Sectorisation is a commonly used technique to increase the wireless networks capacity through spatial reuse of radio resources with typical configuration being a three sectored solution [17].

Unlike conventional network planning in the legacy $<3 \mathrm{GHz}$, higher order sectorisation (HOS) seems a promising key enabler [11][18] to improve network capacity and data throughput, as millimetre wave tends to use highly directional antenna to compensate for the high pathloss at these bands.

In this work, we have modelled five sectorisation orders, namely, 3, 4, 6, 8, and 10 sectors per site, to evaluate their performance in increasing user data rates. Sectorisation can increase network capacity through re-utilising the spectrum in more efficient way, and due to the use of narrow beamwidth in millimetre wave, higher order sectorisation become feasible [11]. HOS advantages will come with increased interference from nearby sectors as clarified in fig.4, where SINR values have been compared among multiple sectorisation orders. This figure shows SINR degradation as the sectorisation order increase. However, beamforming $(\mathrm{BF})$ can greatly reduce this excess interference by generating narrow beams between the base station and UE's to suppress unwanted signals, which eventually increase the SINR [11].

Although, interference slightly increases in HOS scenarios, UE's will receive higher received level due to the directionality of the antennas as the power is concentrated in narrower beamwidth. Furthermore, Reference Signal Received Quality (RSRQ) will deteriorate as a result of increased interference. Fig. 5 shows RSRP improvement in received signal with HOS, which is due to shorter signal path. While the chart on the right shows the degradation in RSRQ with HOS, and this degradation is due to the excess interference. 


\section{Ultra-Dense deployment}

It is widely accepted that current improvements to the LTE system will fall short of meeting the demands of future mobile wireless networks in 2020 and beyond. To meet the $5 \mathrm{G}$ massive demands, network densification is a promising enabler which can be achieved by reducing inter-site-distance (ISD) among small cells. This will insure high path gain to compensate high pathloss at mm-wave, much more physical resources to be used by users, and lower cell load factor. And to satisfy seamless coverage, a large number of small cells have to be densely deployed.

Path gain has been improved when we reduced ISD from scenario A $600 \mathrm{~m}$ to scenario B $400 \mathrm{~m}$ to scenario C $200 \mathrm{~m}$, by deploying 5, 10, and 20 small cells, respectively. Bringing the access point closer to the users will not only ensure high signal strength, but efficient resource utilisation.

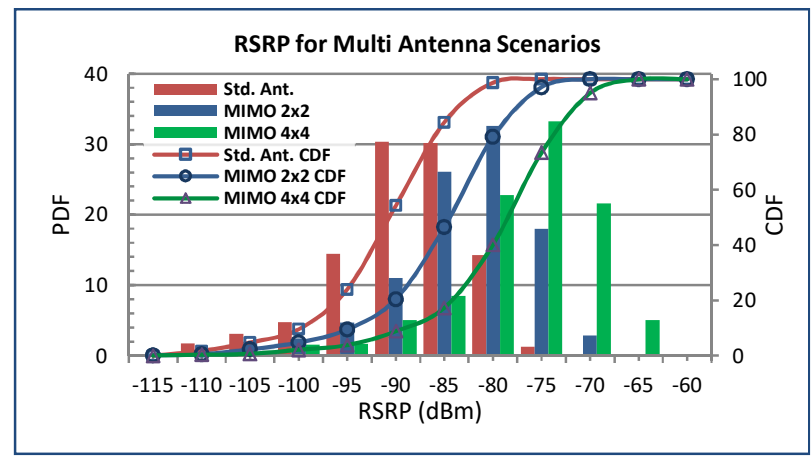

Fig.3 RSRP (dBm) PDF \& CDF distribution for different antennas in mm-wave

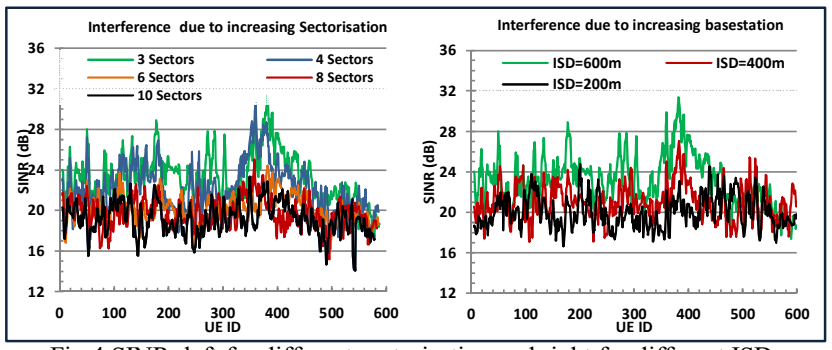

Fig.4 SINR, left for different sectorisation and right for different ISD

However, due to many small cells transmit in specific area, interference is expected to increase as a result. Normally reducing the antennas tilt will reduce the interference by concentrate the power in a more feasible way. Fig.7 clarifies ISD impact on signal level and quality. As per fig.6(a), signal power has been highly improved in scenario C (200m ISD) compared to normal ISD, which highly improves the coverage in this area. Signal quality on the other hand has decreased as a result of increased interference. However, after tilt optimisation, another scenario has been added with tilt optimisation (ScenC-4tilt), RSRQ has improved due to decreased interference (as shown in fig.6(b). Beamforming in dense deployment has a superior performance in term of improving both the signal strength $\&$ quality, since interference is greatly reduced from other direction.

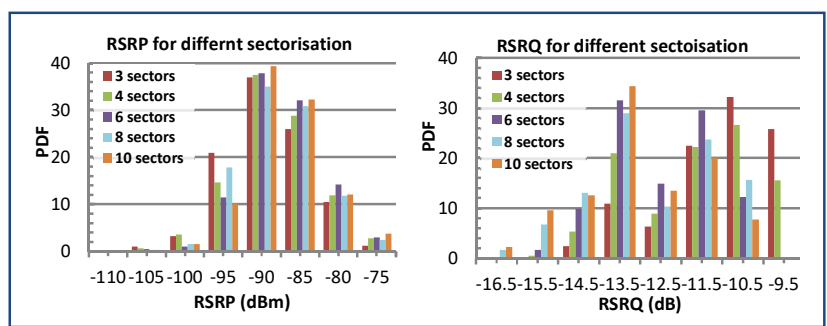

Fig.5 RSRP \& RSRQ for different sectorisation order

\section{Rain Attenuation}

As discussed previously in this paper, rain drops have comparable sizes as the wavelength of mm-wave signal, causing severe attenuation due to scattering. The specific attenuation $(\mathrm{dB} / \mathrm{km})$ increases as the operating frequency increase and as the path length increase as well. Therefore, we have used network densification to reduce rain attenuation at millimetre wave through decreasing ISD. Fig.7 shows the received power before and after heavy rain rate of $50 \mathrm{~mm} / \mathrm{h}$ on different deployment scenarios. As we can see, scenario $\mathrm{C}$ (minimum ISD) performs better and therefore increased the link reliability of millimetre wave network under heavy rain. Nevertheless, an emergency cellular communication is necessary to be available to replace the mm-wave network as heavy rain is likely to disrupt the mm-wave transmission.

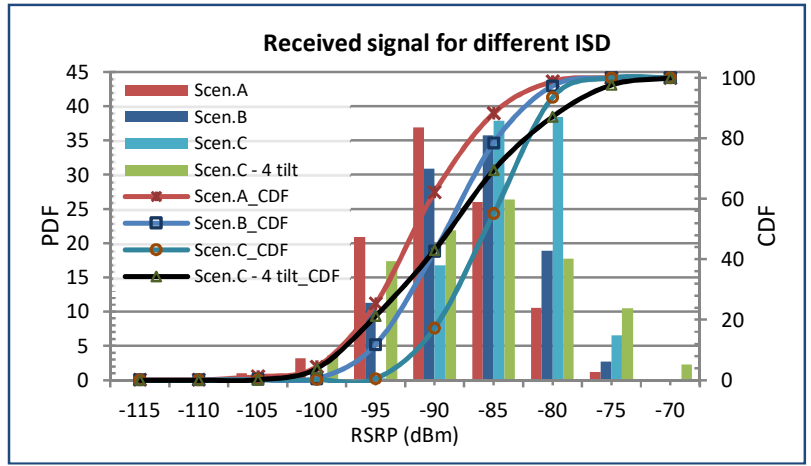

(a)

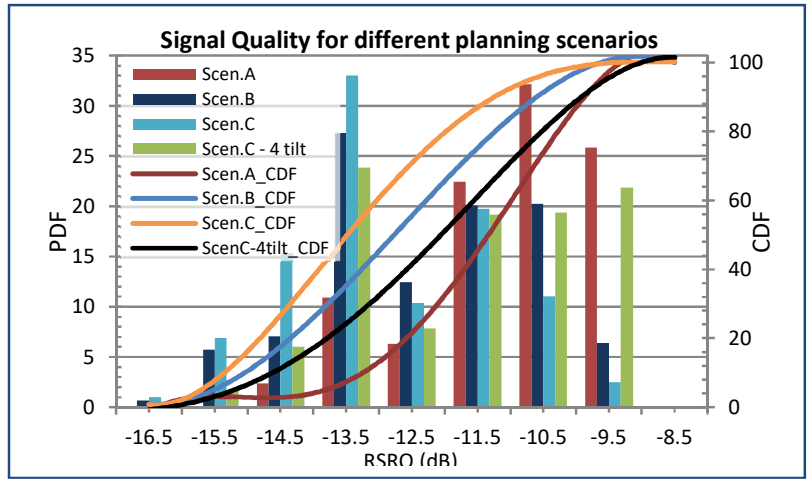

(b)

Fig.6 (a) PDF \& CDF of RSRP (dBm) for ISD 600, 400, $200 \mathrm{~m}$ (b) PDF \& CDF of RSRQ (dB) for ISD $600,400,200 \mathrm{~m}$. 


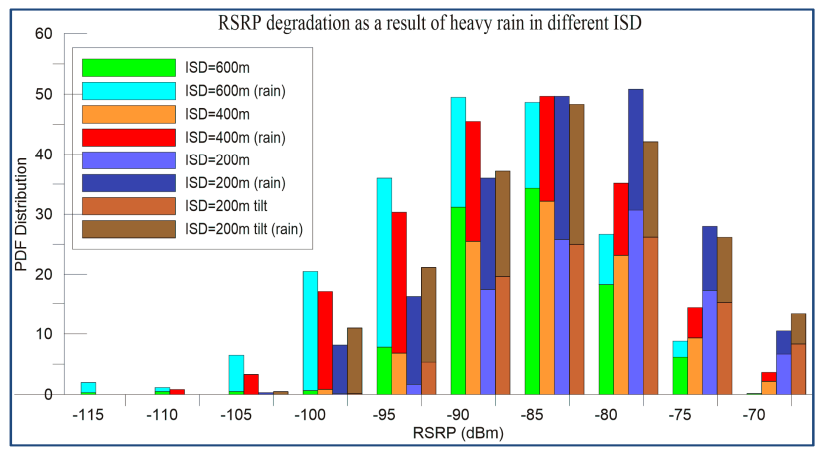

Fig.7 RSRP (dBm) degradation due to heavy rain $(50 \mathrm{~mm} / \mathrm{h})$ for different ISD, clearly scenario-C has less impacted due to shorter path-length.

\section{MASSIVE-MIMO AND BEAMFORMING}

The idea of massive MIMO is the principle when a network is equipped with a very large number of antennas at the base stations, which can accommodate many co-channel users at a time. And beamforming (BF) is the concentration of power in certain direction, which will increase wanted signal power \& suppress other interference efficiently. Beamforming and $\mathrm{m}-$ MIMO are key enabling technologies of next generation $5 \mathrm{G}$ system, as they can significantly improve signal strength, which can lead to higher cell throughput and better cell edge performance compared to $4 \mathrm{G}$ network.

In ultra-dense small cells deployment, hybrid beamforming is more realistic alternative. In this scenario, beamforming weighting is implemented in the RF side, where every group of antennas are stacked together and supplied by single digital-toanalogue convertor (DAC) for beamforming. This hybrid approach can highly reduce the number of RF chains and hardware requirements, and maintain reasonable multiple simultaneous beams for transmission. Beamforming can be used in base station (TxBF) as well as $\mathrm{UE}(\mathrm{RxBF})$, as future mmwave handset could be developed with higher number of antennas that can beam-form and steer their reception and transmission with higher gain and efficiency.

Generally, the gain of increasing the number of antennas is:

$$
G=10 \times \log _{10}(n)
$$

where $n$ is the number of antennas used [18]. In addition to this gain, beamforming has additional gain due to beam directionality (steering gain) which concentrates the power constructively to the desired direction.

A receiver with only four antennas has a standard gain of $6 \mathrm{~dB}$ according to eq.4, an additional gain will be as a result of beamforming directionality (steering the antenna beams to the required destination). The total beamforming gain has highly improved the dense planning.

The improvement comes in term of total interference, as UE's now can reject unwanted interference as shown in fig. $8 \mathrm{a}$; which is showing the interference from all subscribers to all activated base stations. The interference is decreased when $\mathrm{Rx}$ beamforming is enabled to UE's. As a consequence, RSRP and RSRQ have improved with RxBF, as clarified in fig. $8 \mathrm{~b}$.

TxBF has higher gain than RxBF due to the higher number of antennas that can be equipped in the base station. TxBF can simultaneously assign beams to user and improve cell-edge users due to high path gain \& low interference. As shown in fig.9, TxBF outperforms RxBF, which can accommodate higher pathloss and atmospheric attenuation, and improve SINR to fulfil QoE provision in next generation $5 \mathrm{G}$ network.

The data throughput of a user in these scenarios is highly impacted by the interference due to higher densification and small cells density. However, a very high data rates can be expected due to the densification in bandwidth, increase number of sectors and sites, decrease ISD, and beamforming.

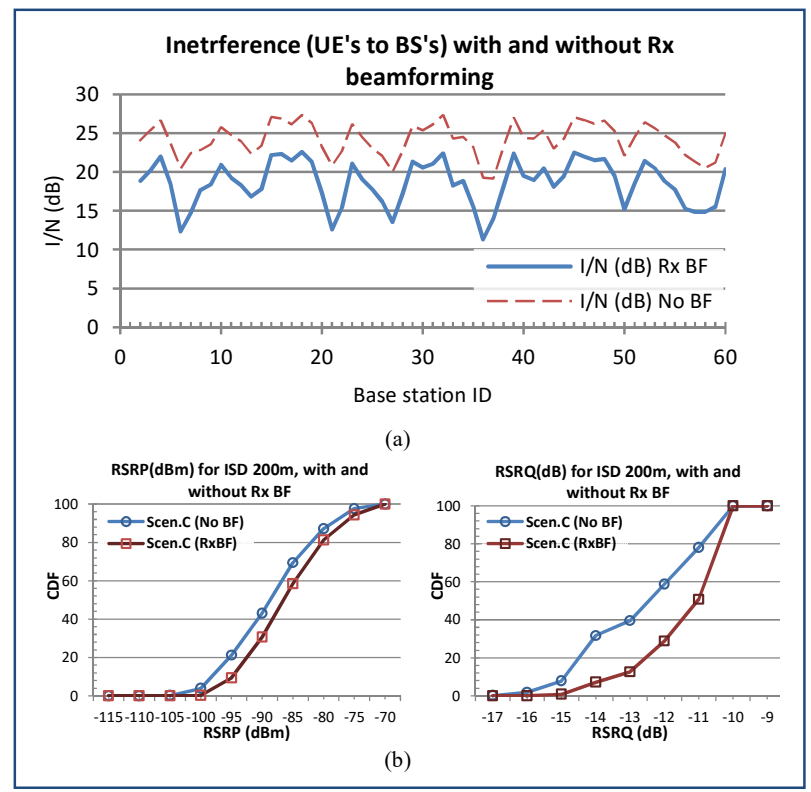

Fig.8 RxBF improvement (a) interference per sector from all subscribers and (b) CDF of RSRP and RSRQ with and without Rx beamforming.

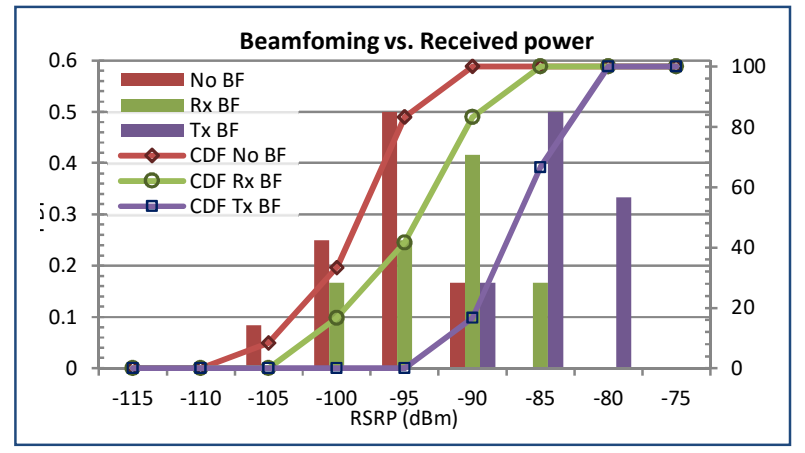

Fig.9 RxBF \& TxBF improvement, showing CDF and PDF of RSRP.

As shown in fig.10, the data throughput has increased as the sectorisation order increased, and this increment is impaired by excess interference. However, when beamforming is applied on ten sectors scenario, where the interference is very high, significant improvement is achieved in data rate. 
Moreover, when interference among sectors is coordinated by inter-cell-interference-coordination techniques in addition to beamforming, higher gain in data throughput can be achieved. Decreasing the ISD through dense station deployment has shown even higher improvement than sectorisation scenarios, as the interference in the former case is quite less as well as it achieved better line-of-site coverage probability. However, sectorisation requires no site acquisition, tower installation, or extra backhauling which make it a simple fast solution. Therefore, a combination of both cases can be consider as a trade-off between installation difficulty and data rate requirements, and the choice will largely depend on the geographical area, the users density, and their demands.

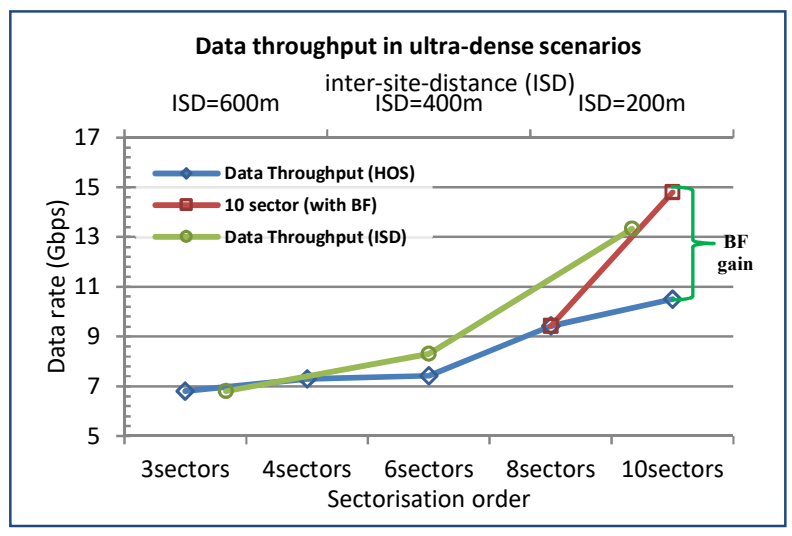

Fig.10 Data throughput (Gbps) for different densification scenarios.

\section{CONCLUSION AND FUTURE WORK}

This paper analyses the feasibility of millimetre wave in $5 \mathrm{G}$ mobile system, which can support multi Gbps data throughput via network densification. The system performance has been evaluated based on received signal power, quality, SINR, and data throughput capability.

In this work, we have introduced a $5 \mathrm{G}$ network that's based on densification, which includes densification over frequency through millimetre wave, and space through higher antennas, higher sectorisation order, and denser deployment of small cells. Our results show that this theme has significantly improved network capacity and user quality of experience, and decrease rain attenuation, which is significant at millimetre wave. In addition, beamforming is a very important in millimetre wave to cope with weak signal transmission and suppress interference. The aforementioned combinations of densification options can efficiently raise the user experience to the level that $5 \mathrm{G}$ vision promised. $5 \mathrm{G}$ will be capable of providing multi Gbps data rates in millimetre wave band and meeting the massive increase in global mobile data traffic. And how to decrease interference in ultra-dense small cells environment in mm-wave will be the topic of future work.

\section{ACKNOWLEDGMENT}

This work is sponsored by the ministry of higher education and scientific research, University of Anbar, Iraq.

\section{References}

[1] Cisco, "Cisco Visual Networking Index: Global Mobile Data Traffic Forecast Update , 2015-2020," White Pap., pp. 1-39, 2016.

[2] F. Boccardi, R. W. H. Jr., A. Lozano, T. L. Marzetta, and P. Popovski, "Five Disruptive Technology Directions for 5G," IEEE Commun. Mag., vol. 52, no. 2, pp. 74-80, 2014.

[3] N. Al-Falahy and O. Alani, "Potential Technologies to 5G Network: Challenges and Opportunities," IEEE IT Professional, vol. 19, no. 1, pp. $12-20,2016$.

[4] F. Khan and Z. Pi, "An Introduction to Millimeter-Wave Mobile Broadband Systems," IEEE Commun. Mag., vol. 59, no. 6, pp. 101-107, 2011.

[5] N. Bhushan et al., "Network Densification: The Dominant Theme for Wireless Evolution into 5G," IEEE Commun. Mag., vol. 52, no. 2, pp. 82 89, 2014.

[6] J. Feng, Z. Feng, Z. Wei, W. Li, and S. Roy, "Optimal Base Station Density in Ultra-densification Heterogeneous Network," in IEEE Wireless Communications and Networking Conference (WCNC): - Track 3: Mobile and Wireless Networks, 2015, pp. 1452-1457.

[7] J. Zhang et al., "Mobility Enhancement and Performance Evaluation for 5G Ultra Dense Networks," in IEEE Wireless Communications and Networking Conference (WCNC), 2015, pp. 1793-1798.

[8] M. Chuang, M. Chang, and Y. S. Sun, "Resource Management Issues in 5G Ultra Dense Smallcell Networks," in International Conference on Information Networking (ICOIN), 2015, pp. 159-164.

[9] S. R. Lamas and D. Gonz, "Indoor Planning Optimization of Ultra-dense Cellular Networks at High Carrier Frequencies," in IEEE Wireless Communications and Networking Conference, 2015, pp. 23-28.

[10] M. N. Kulkarni, S. Singh, and J. G. Andrews, "Coverage and Rate Trends in Dense Urban mmWave Cellular Networks," in Globecom 2014 Wireless Communications Symposium Coverage, 2014, pp. 3809-3814.

[11] N. Al-Falahy and O. Alani, "The Impact of Higher Order Sectorisation on the Performance of Millimetre Wave 5G Network," in The 10th International Conference on Next Generation Mobile Applications. Security and Technologies (NGMAST2016), 2016, pp. 1-5.

[12] ATDI, "ICS Designer by ATDI." [Online]. Available: www.atdi.co.uk/software/radio-planning/ics-designer. [Accessed: 04Apr-2016].

[13] M. R. Akdeniz et al., "Millimeter Wave Channel Modeling and Cellular Capacity Evaluation," IEEE J. Sel. AREAS Commun., vol. 32, no. 6, pp. 1164-1179, 2014.

[14] M. Marcus and B. Pattan, "Millimeter Wave Propagation: Spectrum Management Implications," IEEE Microw. Mag., vol. 6, no. 2, 2005.

[15] F. Khan, Z. Pi, and S. Rajagopal, "Millimeter-wave mobile broadband with large scale spatial processing for $5 \mathrm{G}$ mobile communication," in 50th Annual Allerton Conference on Communication, Control, and Computing (Allerton), 2012, pp. 1517-1523.

[16] C. Sheldon et al., "Spatial multiplexing over a line-of-sight millimeterwave MIMO link: A two-channel hardware demonstration at $1.2 \mathrm{Gbps}$ over $41 \mathrm{~m}$ range," in 1st European Conference on Wireless Technology, 2008, pp. 198-201.

[17] F. Athley, M. N. Johansson, and A. Nilsson, "Increased Sectorization: Horizontal or Vertical ?," in IEEE 78th Vehicular Technology Conference (VTC Fall), 2013, pp. 1-5.

[18] S. Rajagopal, S. Abu-Surra, and F. Khan, "Antenna Array Design for Multi-Gbps mmWave Mobile Broadband Communication," in IEEE Global Telecommunications Conference - GLOBECOM, 2011, pp. 1-6. 